\title{
Labyrinthe
}

39 | 2012 (2)

« Et si... ? » La cause du contrefactuel

\section{Food facts}

\section{Adrien Guignard}

\section{(2) OpenEdition}

Journals

Édition électronique

URL : http://journals.openedition.org/labyrinthe/4283

DOI : $10.4000 /$ labyrinthe.4283

ISSN : 1950-6031

Éditeur

Hermann

Édition imprimée

Date de publication : 31 décembre 2012

Pagination : 153-161

ISBN : 9782705682637

Référence électronique

Adrien Guignard, « Food facts », Labyrinthe [En ligne], 39 | 2012 (2), mis en ligne le 10 janvier 2013

consulté le 20 avril 2019. URL : http://journals.openedition.org/labyrinthe/4283 ; DOI : 10.4000/ labyrinthe.4283

Propriété intellectuelle 


\section{Food facts}

Adrien GUIGNARD

L’homme qui médite est un animal dépravé.

J.-J. Rouseau

Seul l'homme peut être bête de bêtise.

J. Derrida

Me voici seul sur la terre, n'ayant plus de frere, de prochain, d'ami, de société que moi-même. Le plus sociable et le plus aimant des humains en a été proscrit par un accord unanime. ${ }^{1}$

Bien que notre siècle n'ait pas encore débrouillé les paradoxes nouant la solitude superlative du «plus sociable et aimant» des promeneurs resté solitaire, les longs extraits qui suivent voudraient, en cette année du tricentenaire de sa naissance, commencer par répondre favorablement à une question (on la dirait rhétorique) qu'on lira dans la neuvième rêverie. Cette question n'a pas trait à la solitude (nous la retrouverons cependant). Au contraire, elle est adaptée à la circonstance et concerne l'épanouissement festif d'un peuple entier. Plus précisément, il s'agit des «rayons suprêmes du plaisir» dardés sur l'âme sensible, jouissant alors d'un spectacle d'où émane une joie commune, communautaire, communicative : parfaite.

\footnotetext{
1. Toutes les citations de Rousseau proviennent du premier volume (Confessions et autres écrits autobiographiques) des (Euvres complètes, Paris, Gallimard, coll. «Bibliothèque de la Pléiade», 1959. La direction de cette somme d'érudition est celle de B. Gagnebin et M. Raymond. L'édition respecte la graphie originale ainsi que sa ponctuation. Les Rêveries du Promeneur solitaire sont posthumes. $L a$ Première promenade a été rédigée en automne 1776. Quant à la neuvième, éhontément exploitée par mon propos, sa rédaction date de mars 1778. L'étude de J. Starobinski (cf. Largesse, Paris, Réunion des Musées Nationaux, 1994) doit être considérée comme la meilleure interprétation des textes et des contextes que j'agite avec désinvolture.
} 


\section{Labyrinthe, $n^{\circ} 39$}

Est-il une jouissance plus douce que de voir un peuple entier se livrer à la joye un jour de fêtes et tous les cœurs s'épanouir aux rayons suprêmes du plaisir qui passe rapidement mais vivement à travers les nuages de la vie?

À suivre le cours onirique de cette IXe Promenade, c'est pourtant une liesse injuste qui imprègne d'abord la mémoire qui confesse l'épanchement d'un vécu.

Ceci me rappelle un autre amusement à peu près de même espéce dont le souvenir m'est resté de beaucoup plus loin. C'étoit dans le malheureux tems où faufilé parmi les riches et les gens de lettres j'étois quelquefois reduit à partager leurs tristes plaisirs. J'étois à la Chevrette au tems de la fête du maitre de la maison; toute sa famille s'étoit reunie pour la celebrer, et tout l'eclat des plaisirs bruyans fut mis en œuvre pour cette effet. Jeux, spectacles, festins, feux d'artifice, rien ne fut épargné. L'on avoit pas le tems de prendre haleine et l'on s'étourdissoit au lieu de s'amuser. Après le diner on alla prendre l'air dans l'avenue; on tenoit une espéce de foire. On dansoit, les messieurs daignérent danser avec les paysannes mais les Dames gardérent leur dignité. On vendoit là des pains d'epices. Un jeune homme de la compagnie s'avisa d'en acheter pour les lancer l'un après l'autre au milieu de la foule, et l'on prit tant de plaisir à voir tous ces manans se précipiter, se battre, se renverser pour en avoir que tout le monde voulut se donner le meme plaisir. Et pains d'epices de voler à droite et à gauche et filles et garçons de courir, s'entasser et s'estropier; cela paroissoit charmant à tout le monde. Je fis comme les autres par mauvaise honte quoiqu'en dedans je ne m'amusasse pas autant qu'eux. Mais bientôt ennuyé de vuider ma bourse pour faire écraser les gens, je laissai là la bonne compagnie et je fus me promener seul dans la foire.

La compagnie n'était pas bonne et le plaisir (proche du sadisme?), angoissait. C'est qu'il est d'autre moyen de se payer les voluptés du partage.

J'aperçus entre autres cinq ou six Savoyards autour d'une petite fille qui avoit encore sur son inventaire une douzaine de chetives pommes dont elle auroit bien voulu se débarasser. Les Savoyards de leur coté auroient bien voulu l'en debarasser mais ils n'avoient que deux ou 


\section{Food facts}

trois liards à eux tous et ce n'étoit pas de quoi faire une grande bréche aux pommes. Cet inventaire étoit pour eux le jardin des Hesperides, et la petite fille étoit le dragon qui le gardoit. Cette comedie m'amusa longtems; j'en fis enfin le denoument en payant les pommes à la petite fille et les lui faisant distribuer aux petits garçons. J'eus alors un des plus doux spectacles qui puissent flater un cœur d'homme, celui de voir la joye unie avec l'innocence de l'age se répandre tout autour de moi. Car les spectateurs mêmes en la voyant la partagérent, et moi qui partageois à si bon marché cette joye, j'avois de plus celle de sentir qu'elle étoit mon ouvrage.

En comparant cet amusement avec ceux que je venois de quitter je sentois avec satisfaction la différence qu'il y a des gouts sains et des plaisirs naturels à ceux que fait naitre l'oppulence et qui ne sont guéres que des plaisirs de moquerie et des gouts exclusifs engendrés par le mépris. Car quelle sorte de plaisir pouvoit-on prendre à voir des troupeaux d'hommes avilis par la misére, s'entasser, s'etouffer, s'estropier brutalement, pour s'arracher avidemment quelques morceaux de pain d'epice foulés aux pieds et couverts de boue?

De mon côté quand j'ai bien réflechi sur l'espéce de volupté que je goutois dans ces sortes d'occasions j'ai trouvé qu'elle consistoit moins dans un sentiment de bienfaisance que dans le plaisir de voir des visages contens. Cet aspect a pour moi un charme qui bien qu'il penetre jusqu'à mon cœur semble être uniquement de sensation. Si je ne vois la satisfaction que je cause quand même j'en serois sûr je n'en jouirois qu'à demi. C'est même pour moi un plaisir desinteressé qui ne dépend pas de la part que j'y puis avoir.

Si un célèbre épisode des Confessions a pu peindre un Jean-Jacques, alors apprenti graveur chez Abel Ducommun, sous les traits d'un Héraclès chasseur de pommes ${ }^{2}$, le texte cité précédemment met en scène un adulte accompli, guéri des gourmandises et autres cleptomanies de sa jeunesse. Non plus voleur qui se fait pincer par le dragon Ducommun mais, placé en présence d'une jeune et frêle femelle dragon, notre promeneur se met avantageusement en scène. Il tient les cordons de la bourse

2. Cf. éd. citée, p. 34 
et, conséquemment, tire les ficelles ${ }^{3}$ du dénouement ( $«$ j’en fis enfin le denoument en payant»). Le promeneur sait organiser et tirer profit d'une sorte d'innocente vénalité : il jouit alors du «plus doux spectacles qui puissent flater un cœur d'homme, celui de voir la joye unie avec l'innocence de l'age se répandre tout autour de lui». Miam.

Passons un siècle pour citer un Petit poème en prose. Dans ce texte, intitulé Le Gâteau, le narrateur baudelairien - on le dit plus flâneur que promeneur solitaire - pourrait partager avec Rousseau une générosité qui ordonne la charité jusqu'à soi-même, c'est-à-dire à une altitude dont la seule mesure serait une abnégation parfaite, une évaporation. Le Gâteau nous invite, non seulement à voyager vers un «superbe pays» où une parole stupide accomplit une sorte de transsubstantiation du pain en gâteau, mais aussi à constater que l'ontologie miraculeuse du gâteau va et vient de poussière. Ce Gâteau célèbre l'idiotie (au sens étymologique ${ }^{4}$ ) et donne une approximation poétique de ce qu'est la parfaite nature du partage (l'énonciation doublement débile qui lâche "gâteau!», rejoint, par des voies impénétrables, cette «joye unie avec l'innocence de l'age» qu'on vient de lire).

Je voyageais. Le paysage au milieu duquel j'étais placé était d'une grandeur et d'une noblesse irrésistibles. Il en passa sans doute en ce moment quelque chose dans mon âme. Mes pensées voltigeaient avec

3. Cf., éd. citée, p. 1090. N'oublions pas que cette IXe Promenade met également en scène le rêveur (et sa Thérèse) se livrant au truquage d'un jeu d'oublies. Ce beau passage comporte plusieurs strates de significations. Au XVIII ${ }^{\mathrm{e}}$, un oublieur confectionne des petits gâteaux nommés «oublies » qu'il fait tirer au sort sur une roue fichée de billets gagnants ou perdants. Dans la scène que j'évoque, Rousseau soudoie l'oublieur (la nature du marché fera en sorte que la roue ne contienne que des bons lots). Passe un troupeau de jeunes pensionnaires particulièrement hideuses (des défavorisées très moches). Pourtant, régalées par la tricherie généreuse de Rousseau, leur joie fait scintiller leur visage d'une beauté qu'on dira (malgré les ravages du sucre) : authentique. Rousseau n'oublie donc pas que la roue qui destine les hasards des bons lots est perfectible (moyennant finance, «trente sols»). Allégoriquement, en jouant peut-être sur les mots, Rousseau parvient à acheter le Destin et, falsifiant la roue des oublies, les petites filles : «oublie[nt] leur laideur». Quant au lecteur, on ne se sait pas trop ce que le destin lui fait oublier.

4. «Un mot exprime à lui seul ce double caractère, solitaire et inconnaissable, de toute chose au monde : le mot idiotie. Idiôtès, idiot, signifie simple, particulier, unique; puis par une extension sémantique dont la signification philosophique est de grande portée, personne dénuée d'intelligence, être dépourvu de raison. Toute chose, toute personne sont ainsi idiotes dès lors qu'elles n'existent qu'en elles-mêmes, c'est-à-dire sont incapables d'apparaître autrement que là où elles sont et telles qu'elles sont : incapables donc, et en premier lieu, de se refléter, d'apparaître dans le double miroir ». Cf. C. Rosset, Le réel. Traité de l'idiotie, Paris, Minuit, 1997, p. 42. 
une légèreté égale à celle de l'atmosphère [...] mon âme me semblait aussi vaste et aussi pure que la coupole du ciel dont j'étais enveloppé ; le souvenir des choses terrestres n'arrivait à mon cœur qu'affaibli et diminué, comme le son de la clochette des bestiaux imperceptibles [...]. Et je me souviens que cette sensation solennelle et rare, causée par un grand mouvement parfaitement silencieux, me remplissait d'une joie mêlée de peur. Bref, je me sentais, grâce à l'enthousiasmante beauté dont j'étais environné, en parfaite paix avec moi-même et avec l'univers; je crois même que, dans ma parfaite béatitude et dans mon total oubli de tout le mal terrestre, j'en étais venu à ne plus trouver si ridicules les journaux qui prétendent que l'homme est né bon;- quand la matière incurable renouvelant ses exigences, je songeai à réparer la fatigue et à soulager l'appétit causés par une si longue ascension. Je tirai de ma poche un gros morceau de pain [...]. Je découpais tranquillement mon pain, quand un bruit très léger me fit lever les yeux. Devant moi se tenait un petit être déguenillé, noir, ébouriffé, dont les yeux creux, farouches et comme suppliants, dévoraient le morceau de pain. Et je l'entendis soupirer, d'une voix basse et rauque, le mot : gâteau! Je ne pus m'empêcher de rire en entendant l'appellation dont il voulait bien honorer mon pain presque blanc, et j'en coupai pour lui une belle tranche que je lui offris. [...] Mais au même instant il fut culbuté par un autre petit sauvage, sorti je ne sais d'où, et si parfaitement semblable au premier qu'on aurait pu le prendre pour son frère jumeau. Ensemble ils roulèrent sur le sol [...]. Le légitime propriétaire du gâteau essaya d'enfoncer ses petites griffes dans les yeux de l'usurpateur; à son tour celui-ci appliqua toutes ses forces à étrangler son adversaire d'une main, pendant que de l'autre il tâchait de glisser dans sa poche le prix du combat. [...] Le gâteau voyageait de main en main et changeait de poche à chaque instant; mais, hélas! il changeait aussi de volume; et lorsque enfin, exténués, haletants, sanglants, ils s'arrêtèrent par impossibilité de continuer, il n'y avait plus, à vrai dire, aucun sujet de bataille; le morceau de pain avait disparu, et il était éparpillé en miettes semblables aux grains de sable auxquels il était mêlé.

Ce spectacle m'avait embrumé le paysage, et la joie calme où s'ébaudissait mon âme avant d'avoir vu ces petits hommes avait totalement disparu; j'en restai triste assez longtemps, me répétant sans cesse : «Il 
y a donc un pays superbe où le pain s'appelle du gâteau, friandise si rare qu'elle suffit pour engendrer une guerre parfaitement fratricide ${ }^{5}$ !

Le Gâteau permet de nombreuses lectures (et justifierait presque le partage très problématique d'une impossibilité de lecture : Le Gâteau et/ou pain a en effet disparu...). Certaines analyses complexes (cf. note 5) insistent sur les paradoxes d'un titre dévidant la fantastique construction de la disparition de ce qu'il intitule pourtant et, par la figure (monstrueuse) du même, rend possible un échange aussi impossible que féroce : un échange (d')abruti(s). D'autres liront évidemment le journal pour analyser l'ironie baudelairienne à l'endroit du promeneur solitaire. Au passage, ils noteront que la gradation qui étage un paysage «état de l'âme» est admirablement construite. L'échange entre les propriétés paysagères et celles du moi s'organise avec une progression repérable («quelque chose» du paysage «passe[rait] dans l'âme»/《 les pensées » gagnent «l'atmosphère »/l'âme devient «la coupole du ciel»/la beauté qui environne le moi transite et rend celui-ci «en parfaite paix avec l'univers»). Bref, nous voici dans un paroxysme de sublime atmosphérique, céleste et universel. L'intertexte journalistique est patent : le moi qui communie en «parfaite béatitude » avec la nature sublime, «dans [son] total oubli de tout le mal terrestre, en [est] venu à ne plus trouver si ridicules les journaux qui prétendent que l'homme est né bon ». Il y aurait encore beaucoup à dire du réemploi ironique de la IXe Promenade, Le Gâteau prend en effet le contre-pied de la dénonciation rousseauiste (avec Baudelaire nous devrions prendre plaisir au spectacle «d'hommes avilis» qui «s'entassent, s'étouffent, s'estropient brutalement, pour s'arracher avidement quelques morceaux de pain $[. .$.$] foulés aux pieds et couverts de boue»).$

\footnotetext{
5. C. Baudelaire, «Le Gâteau», dans Le Spleen de Paris (XV). «Le Gâteau» paraît en 1862 dans La Presse. L'édition Gallimard, coll. «Bibliothèque de la Pléiade» (1990) relève (p. 1319) que le petit lac du Gâteau est «celui d'Escoubous, au-dessus de Barèges», dans les Pyrénées. Les savants austères, amoureux fervents des lectures référentielles apprendront dans le livre de J. Fourcassié, $L e$ Romantisme et les Pyrénées, (Paris, Gallimard, 1940) que Baudelaire a voyagé avec son beau-père dans les Pyrénées. L'existence des «petits êtres » déguenillés n'est, à ma connaissance, pas enregistrée à l'état civil. B. Johnson a proposé une étude «déconstrutrice» de quelques Petits Poèmes en prose (notamment celui-ci,p. 142 et suiv.). Cf. Défiguration du langage poétique, Paris, Flammarion, 1979. Notons que le «conflit des doubles, l'impossibilité de différencier les frères ennemis» fait l'objet d'une partie (Violence et comparaison) du beau livre de J. Thélot, Baudelaire : violence et poésie, Paris, Gallimard, 1992.
} 


\section{Food facts}

Toutefois, je ne crois pas que la réhabilitation du plaisir sadique soit prioritaire. Soyons plus simple et idiot. Ne pourrait-on pas soutenir que le «superbe pays » dont le narrateur baudelairien «répète sans cesse» la déduction n'est pas différent de celui que l'incipit de la première promenade met en place : «Me voici seul sur la terre, n'ayant plus de frere, de prochain, d'ami, de société que moi-même » ? N'est-ce pas là une syntaxe «parfaitement fratricide»?

Ayant laborieusement réconcilié la promenade solitaire avec l'amer savoir du voyage, je voudrais faire partager au lecteur la bonne part du gâteau, ou plutôt d'une tartine. Le point de vue sera plus statique. Le spectateur représenté, mal en point, ne voyage pas ; on le sait splénétique et borderline. Il s'est trainé péniblement dans son jardin de Fontenay-auxRoses et, par-dessus «la haie», observe la route. La citation d'À rebours (le chef-d'œuvre de J.K. Huysmans paraît en 1884) qui suit n'entend pourtant rien de moins que viser la pleine harmonie avec notre contexte social. Tout se passe comme si la Vérité se trouvait naturellement $\grave{A}$ rebours. Dans la scène qu'on va lire, le désir - toujours mimétique -, va bien exactement à rebours. Le pauvre met cyniquement en appétit le dandy «fin de race» (Des Esseintes). Il ne s'agira ni de soutenir que l'homme naît bon par nature, ni de sans cesse répéter les paradoxes d'un échange impossible. Il s'agit simplement de dire la vérité. Celle-ci demeure simple. La violence est le fait premier, la donnée originelle (Food facts... ce sont ces mathématiques marketeuses de brique de lait qui doivent nous dicter les usages partagés du bien). Il n'est somme toute pas besoin de faire intervenir le moindre don, qu'il soit pervers ou charitable, pour que la violence prenne naissance. C'est probablement ce que nous fait comprendre Des Esseintes, tiraillé par les «névroses gastriques ». La lutte entre les enfants a commencé avant que Des Esseintes n'en soit devenu le spectateur (les enfants ne verront jamais Des Esseintes, d'ailleurs). Puissent les éléments du texte qui clora mon propos justifier la «cruelle et abominable loi de la lutte pour l'existence» (ou darwinisme social). Nous saurons, avec la distance qui sied, enseigner ce message «fin de siècle » aux enfants du troisième millénaire. Soyons sans pitié : mieux vaut accélérer le mal et la destruction. Puissent les mères de notre siècle ne «mettre bas» qu'une élite éclairée.

[...Des Esseintes] examina le jardin, s'intéressant aux plantes flétries par la chaleur, et aux terres ardentes qui fumaient dans la pulvérulence 
embrasée de l'air; puis, au-dessus de la haie séparant le jardin en contrebas de la route surélevée montant au fort, il aperçut des gamins qui se roulaient, en plein soleil, dans la lumière. Il concentrait son attention sur eux quand un autre, plus petit, parut, sordide à voir; il avait des cheveux de varech remplis de sable, deux bulles vertes audessous du nez, des lèvres dégoûtantes, entourées de crasse blanche par du fromage à la pie écrasé sur du pain et semé de hachures de ciboule verte. Des Esseintes huma l'air; un pica, une perversion s'empara de lui; cette immonde tartine lui fit venir l'eau à la bouche. Il lui sembla que son estomac, qui se refusait à toute nourriture, digèrerait cet affreux mets et que son palais en jouirait comme d'un régal. Il se leva d'un bond, courut à la cuisine, ordonna de chercher dans le village une miche, du fromage blanc, de la ciboule, prescrivit qu'on lui apprêtât une tartine absolument pareille à celle que rongeait l'enfant, et il retourna s'asseoir sous son arbre. Les marmots se battaient maintenant. Ils s'arrachaient des lambeaux de pain qu'il s'enfonçait, dans les joues, en se suçant les doigts. Des coups de pied et des coups de poing pleuvaient et les plus faibles, foulés par terre, ruaient, et pleuraient, le derrière raboté par les caillasses. Ce spectacle ranima Des Esseintes; l'intérêt qu'il prit à ce combat détournait ses pensées de son mal; devant l'acharnement de ces méchants mômes, il songea à la cruelle et abominable loi de la lutte pour l'existence, et bien que ces enfants fussent ignobles, il ne put s'empêcher de s'intéresser à leur sort et de croire que mieux eût valu pour eux que leur mère n'eût point mis bas. [...]. Le domestique interrompit les charitables réflexions que ruminait Des Esseintes, en lui apportant sur un plat de vermeil la tartine qu'il avait souhaitée. Un haut de cœur le tordit [...]. - Jetez cette tartine, ditil au domestique, à ces enfants qui se massacrent sur la route; que les plus faibles soient estropiés, n'aient part à aucun morceau et soient, de plus, rossés d'importance par leurs familles quand ils rentreront chez elles les culottes déchirées et les yeux meurtris; cela leur donnera un aperçu de la vie qui les attend ${ }^{6}$ !

Certains moments de l'agencement proposé sont légers. D'autres plus graves. Je pense ici aux propos tenus aux portes de l'idiotie. Je n'exclus pas qu'une bêtise («celle qui donne aux yeux cette limpidité morne des

6. J. K. Huysmans, À rebours [1884], Paris, Robert Laffont, coll. «Bouquins», 2005, p. 717 et suiv. 
étangs noirâtres, et ce calme huileux des mers tropicales ») ait pu agacer. On sait depuis les travaux ${ }^{7}$ de Barthes, Deleuze, Ronell, Derrida, Roger, Coste et Rosset que les mots «bête», « idiot», « stupide», sont aussi complexes qu'embrouillés. D'autre part leur violence (ils frappent de stupeur l'exercice de la raison humaine) paraît - avec vanité - s'essayer à repenser l'humanisme. L'importance du droit des animaux (de nos amies les bêtes) aurait-elle «instinctivement» partie liée avec celle du droit des humaines, des humains, des enfants (de genre féminin et de genre masculin)? Laissons les bêtes et les enfants. Oserais-je être plus «politique» et avoir la correction moins allusive de contredire le mot de Brassens? «Le temps fait en effet quelque chose à l'affaire». Pour ceux que l'intelligibilité funambulesque de ce qui précède auraient fâchés, je voudrais poser que les trois textes glosés ne tressent pas un répertoire de paralogismes allusifs et saugrenus. Ils permettront au lecteur de construire une fable, fable de l'évolution, ou plutôt du néolibéralisme. S'il m'appartenait d'en livrer la morale, elle prendrait la forme assertive qui suit. Nous tous, intimement rousseauistes, sommes devenus des Des Esseintes par dressage.

*

Adrien Guignard a soutenu une thèse en littérature française intitulée "Impressions » ironiques du voyage en Suisse et dans les Alpes à l'université de Lausanne. Il est membre du Groupe de la Riponne. Parmi ses quelques publications, celle qui suit pourrait avantageusement éclairer la présente contribution : "Sokal et Bricmont sont sérieux ou : le chat est sur le paillasson», Multitudes, 31 (2007), p. 123-131.

7. Sur l'importance de la «bêtise» comme forme étrange et inquiétante d'un humanisme «à rebours », on lira avec profit. J. Derrida, Séminaire. La bête et le souverain : Volume I (2001-2002), Paris, Galilée, 2008. Il s'agit d'une publication posthume. Les éditeurs sont : M. Lisse, M.-L. Mallet, G. Michaud. Puis, le livre de son élève : A. Ronell, Stupidity, Paris, Stock, 2006 [2001]. La traduction est de C. Surprenant. On ajoutera : A. Roger, Bréviaire de la bêtise, Paris, Gallimard, 2008. Ainsi que : C., Coste, Bêtise de Barthes, Paris, Klincksieck, coll. «Hourvari», 2011. Enfin, renvoyons à : G. Deleuze, Différence et répétition, Paris, PUF, coll. «Epiméthée», p. 197 et suiv. 